\title{
Action de l'arséniate d'étain sur divers cestodes du mouton
}

\author{
par P. CASTEL, M. GRABER, G. GRAS et CHHAY-HANCHENG
}

Le téniasis ovin constitue dans les zones d'élevage de la République du Tchad une affection très répandue et assez grave. Environ 63 p. 100 des moutons de ces régions hébergent des Anoplocephalidae divers (Moniezia expansa, Moniezia benedeni, Stilesia globipunctata, Stilesia hepatica et Avitellina centripunctata), seuls ou associés.

La lutte contre ces cestodes n'est pas facile, car l'action des ténifuges classiques (sulfate de cuivre ; camala ; N. C. A., etc...) se révèle décevante : étant le plus souvent monovalents, ils permettent la destruction totale de Moniezia exponsa et de Moniezia benedeni, sans pouvoir assurer intégralement celle de Stilesia globipunctata, de Stilesia hepatica ou d'Avitellina centripunctata.

Aussi, depuis quelques années, avons-nous cherché à connaître la valeur de corps plus polyvalents capables d'éliminer d'un seul coup tous les cestodes du mouton. Notre attention s'est portée sur un certain nombre d'anthelminthiques et plus particulièrement sur les arséniates métalliques.

\section{A) HISTORIQUE}

Le premier en date, l'arséniate de plomb $A s \mathrm{O}^{4} \mathrm{HPb}$, est utilisé depuis fort longtemps en agriculture comme antiparasitaire. II est de ce fait susceptible d'intoxiquer accidentellement les animaux venus pâturer au voisinage des zones traitées. De nombreux travaux ont été publiés à ce sujet en Amérique (Seddon et Ramsay, 1933 ; Mc Culloch et St John, 1940 ; Blaxter, Allcroft et Ruth, 1950), en Angleterre (Adams et Hugues, 1950), en Australie (Hutchins, 1955), en France (Canat, 1956), en Allemagne (Remy, 1956 ; Schoberl, 1958) et en Hollande (Hoskam, 1958). Le versenate de calcium

Reçu pour publication : mars 1960.

Rev. Elev. Méd. Pays trop., no 1, 13, 1960. ou Ca E. D. T. A. ou edathamil-calcium disodique serait actuellement le meilleur antidote de I'intoxication par le plomb (Lewis et Meikle, 1956).

En médecine vétérinaire, à la suite des travaux de Mc Culloch ef de Mc Cloy (1941), l'arséniate de plomb est considéré comme un bon ténifuge. De très nombreux essais effectués pour la plupart en Amérique ont confirmé la grande efficacité du produit sur Moniezio exponsa, et plus récemment au Tchad, sur Stilesia globipunctata et Avitellina centripunctata du mouton (Graber, 1957):

Malheureusement, le composé reste un toxique dangereux. Le plomb en est vraisemblablement le plus grand responsable, car ce métal a des effets qui pour être moins spectaculaires que ceux de l'arsenic, n'en sont pas moins plus insidieux, tant chez l'animal traité que chez l'homme qui manipule le produit. De plus, l'action anthelminthique du plomb n'a jamais été étudiée à notre connaissance. Enfin on ne possède que fort peu de renseignements sur les quantités de plomb déposées dans les muscles et les organes des moutons soumis au traitement à l'arséniate de plomb.

Aussi a-t-il été abandonné au Laboratoire de Farcha ef les investigations se sont orientées alors vers d'autres arséniates métalliques. Déjà les Russes s'étaient engagés sur la même voie, le «Filixan» et le sulfate de cuivre ne leur donnant pas entière satisfaction. Akramovski et ses collaborateurs (1957), ont «testé 》 sept arséniates métalliques sur le mouton : arséniate d'aluminium, d'étain, de fer (bi et trivalent), de zinc, de calcium et de cuivre. Ils ont obtenu de bons résultats sur Moniezia expansa du mouton à des doses allant de 0,3 à $0,5 \mathrm{~g}$ par tête. Par la suite, Chubabriya (1958) rajoute l'arséniate de manganèse.

Seul de tous ces composés, l'arséniate d'étain présentait a priori le plus de possibilité : l'étain 
est doué en effet d'un pouvoir ténifuge certain (Gras, 1956 ; Castel, Harant et Gras, 1958 a et b). D'autre part, les composés minéraux de l'étain sont très faiblement absorbés dans le tube digesif (Gras, 1956, Barnes et Stoner, 1959) ; leur toxicité est pratiquement nulle. Aussi, la présence éventuelle d'étain dans la viande de boucherie ne pose pas de problème.

C'est effectivement ce qui a eu lieu. En U. R. S. S., les premiers essais remontent à 1955 (Chubabriya). Par la suite, l'arséniate d'étain a été expérimenté par de nombreux chercheurs (Garkavi, 1956 ; Ulyanov, 1957 ; Chubabriya, 1957 ; Grigoryan, 1958 ; Boev et Orlov, 1958 ; Chubabriya 1958 et 1959 ; Paskalskaya, 1959) et en Chine (Anon. 1958). En France, Castel ef Gras (1959), comparant l'action de l'arséniate de plomb et de l'arséniate d'étain sur Hymenolepis fraterna de la souris, ont mis au point une méthode permettant de procéder à un tri préclable parmi les anthelminthiques que l'on désire essayer dans la lutte contre le téniasis ovin. Par la suite, 10 arséniates métalliques ont été essayés chez la souris, les conclusions de cette étude sont les suivantes (Castel, Gras, Graber et Chhay Hancheng 1960). Seul l'arséniate de zinc est plus actif que l'arséniate d'étain. Ce composé sera mis en expérience prochainement.

\section{B) CARACTÉRISTIQUES DE L'ARSÉNIATE D'ÉTAIN}

L'arséniate d'étain employé a été préparé à la Faculté de Pharmacie de Montpellier. Les méthodes de préparation * ef de contrôle du produit seront publiées prochainement (Castel, Gras, Graber et Chhay Hancheng,1960).

Le produit utilisé est l'arséniate monoacide stanneux de formule $\mathrm{AsO}_{4} \mathrm{HSn}, \frac{1}{2} \mathrm{OH}_{2}$. II se présente sous forme d'une poudre fine, blanche et amorphe, insoluble dans l'eau et dans les acides nitrique ef sulfurique dilués; mais soluble dans l'acide chlorhydrique et dans l'acide sulfurique concentré et chaud. II est très soluble dans l'ammoniaque, la soude ef la potasse. II ne présente pas de point de fusion caractéristique. L'arsé-

(*) Laboratoire de pharmacie chimique. - Professeur Castel, Brevet français, no 1642. niate d'étain doit être conservé dans un endroit sec à l'abri de la lumière, dans un flacon hermétiquement clos, de préférence coloré. A l'air libre le produit brunit très légèrement ce qui correspond à une oxydation qui se traduit par une baisse de l'étain stanneux et une augmentation de l'étain stannique. Ces précautions prises la durée de conservation serait pratiquement illimitée (Chubabriya, 1959). En tous cas, l'arséniate d'étain entreposé depuis près de onze mois au laboratoire de Farcha (Tchad) n'a rien perdu de ses qualités anthelminthiques et ne paraît pas avoir subi de modifications physiques sensibles.

Il existe peu de renseignements sur les arséniates d'étain dans la littérature. Prasad (1939) a décrit sommairement un arséniate stannique dans une étude consacrée à la formation des gels. Plus récemment, Sigeto Hamada (1949) s'est efforcé de préciser le pouvoir insecticide de l'arséniate stanneux par rapport à celui des arséniates de plomb et de calcium.

Quant à l'arséniate d'étain mis au point par les chercheurs russes de l'Institut d'élevage et de médecine vétérinaire de Géorgie, il se rapproche singulièrement du nôtre (Chubabriya, 1958 et 1959). Sa formule serait $A s \mathrm{O}^{4} \mathrm{HSn}, \mathrm{H}^{2} \mathrm{O}$, arséniate stanneux. Il s'agit d'une poudre amorphe, de coloration blanche, sans la moindre odeur ou goût spécifique, insoluble dans l'eau et les acides, mais parfaitement soluble dans les alcalis (Chubabriya, 1958).

\section{C) MATÉRIEL ET MÉTHODE}

Les essais ont eu lieu de mai 1959 à février 1960 c'est-à-dire pendant un laps de temps englobant la fin de la saison sèche (mai, juin, juillet), la saison des pluies (de juillet à octobre) et le début de la saison sèche suivante (octobre à février), ce qui a permis de suivre le comportement des animaux traités en fonction des diverses saisons.

Les" 126 moutons mis en expérience venaient de la région ovest du Tchad;: leur état d'entretien était dans l'ensemble médiocre, surtout en fin de saison sèche et au cours de la saison des pluies. Ils hébergeaient tous des Anoplocephalidae, seuls ou associés, appartenant aux espèces suivantes : Moniezia expansa, Moniezia benedeni, Stilesia globipunctata, Stilesia hepatica, Avitellina 
centripunctato et Avitellino woodlandi (un cas). Ces cestodes en nombre souvent important, se trouvaient être à des stades de développement différents, ou très jeunes, ou adultes rejetant des anneaux dans les excréments.

Chaque animal a été assujetti à un contrôle rigoureux pendant 10 jours : deux jours de stabulation préalable avec mise en évidence des anneaux de Moniezia et d'Avitellina, de manière à éliminer les non-porteurs; traitement, puis mise en observation de huit jours pendant lesquels les crottes ont été ramassées, broyées dans de l'eau et minutieusement examinées dans le but de prélever les fragments d'Anoplocephalidae présents. Passé ce temps, les moutons ont été abattus ef l'intestin visité complètement. Les scolex de Moniezia et d'Avitellina ont été recherchés avec soin. Les nodules de Stilesia, au voisinage du duodénum, ont été soumis à un grattage profond, avec étalement du produit de raclage entre lame et lamelle et examen à l'état frais au microscope, les scolex de Stilesia devant être facilement reconnus.

Les fragments de cestodes recueillis dans les excréments après traitement et ceux récoltés dans l'intestin après autopsie, ont été pesés séparément. La comparaison entre ce qui est évacué (en grammes) après traitement ef ce qui reste permet d'apprécier l'efficacité du produit, compte tenu bien entendu, des résultats fournis par le grattage des muqueuses.

\section{D) RÉSULTATS}

Nous avons procédé par tâtonnement en étudiant l'influence de certains facteurs (diète, administration d'arséniate en poudre ou en capsules).

TABLEAU No 1

Administration d'arséniate d'étain mélangé à de l'eau, en une fois, sans diète préalable

\begin{tabular}{|c|c|c|c|c|c|c|}
\hline $\begin{array}{l}\text { Doses } \\
(\text { par tête) } \\
\text { en mg. }\end{array}$ & $\begin{array}{c}\text { Nombre } \\
\text { d'animaux }\end{array}$ & Poids (kg) & Parasites en cause & $\begin{array}{l}\text { Efficacit } \\
\text { Extensive } \\
\text { E.E. }\end{array}$ & $\begin{array}{l}\text { té }(\boldsymbol{C}) \\
\text { Intensive } \\
\text { I.E. }\end{array}$ & Mortalité \\
\hline 200 & $\begin{array}{l}3 \\
1 \\
1\end{array}$ & $\begin{array}{l}14,20,21 \\
20 \\
21\end{array}$ & $\begin{array}{c}\text { Moniezia expansa } \\
\text { Stilesia globipunctata } \\
\text { Avitellina centripuratata }\end{array}$ & $\begin{array}{r}66 \\
0 \\
100\end{array}$ & $\begin{array}{r}96 \\
0 \\
100\end{array}$ & 1 \\
\hline 250 & $\begin{array}{l}4 \\
1 \\
2 \\
3\end{array}$ & $\begin{array}{c}25,15,17,12 \\
25 \\
25,15,17,12\end{array}$ & $\begin{array}{l}\text { Moniezla axpansa } \\
\text { Stilesia globipunctata } \\
\text { Stilesia hepatica } \\
\text { Avitelling centripunctata }\end{array}$ & $\begin{array}{r}100 \\
0 \\
0 \\
100\end{array}$ & $\begin{array}{r}100 \\
0 \\
0 \\
100\end{array}$ & 1 \\
\hline 300 & $\begin{array}{l}2 \\
1\end{array}$ & ${ }_{19}^{19} 19$ & $\begin{array}{l}\text { Momiesia expansa } \\
\text { Avitelling centripunctata }\end{array}$ & $\begin{array}{l}100 \\
100\end{array}$ & $\begin{array}{l}100 \\
100\end{array}$ & 1 \\
\hline 400 & $\begin{array}{l}1 \\
2\end{array}$ & 16,19 & $\begin{array}{l}\text { Stilesia hepatica } \\
\text { Avitellina centripuctata }\end{array}$ & $\begin{array}{r}0 \\
100\end{array}$ & $\begin{array}{r}0 \\
100\end{array}$ & \\
\hline 500 & $\begin{array}{l}3 \\
3\end{array}$ & $\begin{array}{l}16,19,15 \\
15,15,19\end{array}$ & $\begin{array}{l}\text { Moniezia expansa } \\
\text { Afitellins oentripuctata }\end{array}$ & $\begin{array}{l}900 \\
100\end{array}$ & $\begin{array}{l}100 \\
100\end{array}$ & 2 \\
\hline 1.000 & $\begin{array}{l}2 \\
2 \\
3\end{array}$ & $\begin{array}{c}17,18 \\
17,20 \\
17,18,20\end{array}$ & $\begin{array}{c}\text { Kaniezia expansa } \\
\text { Stilesia globipunctata } \\
\text { Avitellina centripunctata }\end{array}$ & $\begin{array}{l}100 \\
100 \\
100\end{array}$ & $\begin{array}{l}100 \\
100 \\
100\end{array}$ & 2 \\
\hline 2.000 & $\begin{array}{l}2 \\
1 \\
2\end{array}$ & $\begin{array}{l}16,18 \\
19 \\
16,19\end{array}$ & $\begin{array}{c}\text { Moniezia expansa } \\
\text { Stilesia globipunctata } \\
\text { Avitellina centripunctata }\end{array}$ & $\begin{array}{l}100 \\
100 \\
100\end{array}$ & $\begin{array}{l}100 \\
100 \\
100\end{array}$ & 3 \\
\hline
\end{tabular}


1) Premier temps : pas de diète. - L'arséniate d'éłain en poudre est administré en une seule fois mélangé à de l'eau dans une bouteille.

II serait trop long de donner les résultats individuels et nous nous contenterons des deux coefficients statistiques proposés par R. S. Schultz, à savoir :

L'efficacité extensive (E. E.) ou pourcentage d'animaux totalement débarrassés de leurs parasites.

L'efficacifé intensive (I. E.) ou pourcentage de réduction de la quantité de parasites après administration de l'anthelminthique.

Les résultats sont consignés au tableau I.

Deux constatations s'imposent :

a) Sur le parasite, on remarquera le haut degré d'efficacité de l'arséniate d'étain sur Moniezia expansa et Avitellina centripunctata, à partir de $250 \mathrm{mg}$ par tête ; par contre, Stilesia hepatica est retrouvé intact dans les canaux biliaires de son hôte. Stilesia globipunctata paraît plus résistant que Moniezia ou Avitellina, sans qu'il soit possible, à la suite de ce premier essai, de déterminer exactement la dose d'arséniate d'étain suffisante pour détruire ce cestode.

b) Sur le mouton, la toxicité de l'arséniate d'étain administré sous forme de poudre est réelle, que la dose soit faible $(200 \mathrm{mg})$ ou forte $(2 \mathrm{~g})$. Tous les moutons morts présentaient les mêmes symptômes en plus ou moins accusés suivant l'importance de la dose reçue : inappétence ; tristesse; soif intense; pas de coliques; diarrhée séreuse, profuse dans un certain nombre de cas. L'animal reste couché ef maigrit considérablement. La mort survient de 50 à 80 heures après le traitement.

A l'autopsie, les principales lésions rencontrées siègent sur l'appareil digestif, notamment sur les premières portions; on note une inflammation aiguë de la caillette avec piqueté rouge sur toute sa surface. L'intestin est congestionné, mais il y a rarement hémorragie. Quelquefois, surtout avec les doses les plus élevées, l'organe semble complètement « décapé » ; transparent, il s'en va par lambeaux.

Le poumon et le foie sont normaux. On observe également quelques cas de néphrite.

FIG. 1. COURBB DB I'ARSENIC SOLUBLF

DE L'ARSENIATE DIBTAIN BN FORCTION DU pH.

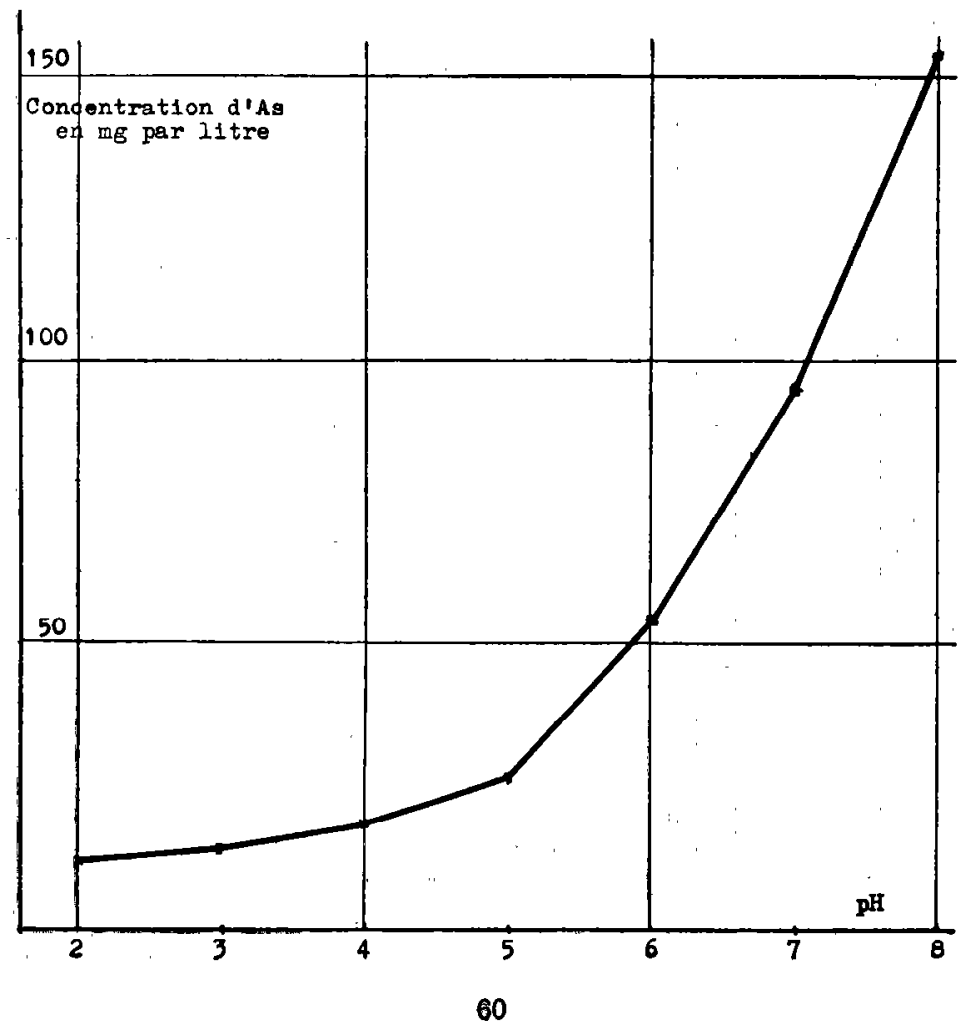


Ces résultats sont en contradiction formelle avec ceux obtenus par Chubabriya (1958), nous avons tenté d'éclaircir ce point. Nous nous sommes penchés spécialement sur le problème des variations « de l'arsenic soluble » de l'arséniate d'étain en fonction du $\mathrm{pH}$. On sait que l'analyse de l'arsenic soluble est une détermination qui est régulièrement faite dans le contrôle des insecticides arsenicaux agricoles. Nous avons étudié cette question avec une technique un peu différente qui nous a semblé plus en rapport avec les conditions physiologiques. La technique utilisée peut être résumée de la manière suivante. Un excès d'arséniate d'étain est délayé dans un bécher avec $50 \mathrm{ml}$ d'eau distillée à des $\mathrm{pH}$ déterminés préalablement par la méthode électrométrique. Le bécher est placé pendant 1 heure à l'étuve à $37^{\circ}$ et la suspension soumise à une agitation constante pendant tout ce temps. Après un repos de 10 minutes on filtre et l'arsenic est dosé dans la solution par la méthode spectrophotométrique de Cristau (1958) utilisant le réactif de Bougault. Les résultats sont rapportés sur le graphique (fig. 1). Chaque point de la courbe est la moyenne d'au moins 3 dosages.

Ces résultats montrent que l'arséniate stanneux se comporte comme tous les sels d'étain et qu'il est hydrolysé fortement pour les $\mathrm{pH}$ voisins de la neutralité. Cette hydrolyse s'accompagne de la formation d'hydroxyde d'étain insoluble avec libération d'anhydride arsénique soluble. L'anhydride arsénique passe dans la circulation générale et intoxique l'animal.

Or le $\mathrm{pH}$ de l'eau de forage du laboratoire était de 6,4. En procédant selon la manière indiquée c'est-à-dire administration du produit dans de l'eau, une certaine fraction de l'arséniate d'étain s'est trouvé solubilisée dès le départ et s'est ajoutée à celle normalement libérée au confact des sucs stomacaux ( $\mathrm{pH}$ 6,5-6,6) et intestinaux $(\mathrm{pH} 7,8-8,1)$, d'où excès d'arsenic soluble et mort de l'animal par intoxicalion arsenicale. Trois dosages effectués sur des moutons ayant absorbé deux grammes d'arséniate en apportent la preuve (tableau II).

L'arsenic a été dosé par la méthode de Cribier (1921) et Griffon (1933), l'étain par la méthode spectrophotométrique au dithiol d'Ovenstone et Kenyon (1955).

Pour remédier à cet état de chose, et limiter la production d'arsenic soluble, il a été décidé
TABLEAU No II

Teneur en étain et arsenic de différents organes de moutons morts par intoxication arsenicale

\begin{tabular}{|c|c|c|c|}
\hline & Organes & As en $\mathrm{mg} / \mathrm{kg}$ & $\mathrm{Sn}$ en $\mathrm{mg} / \mathrm{kg}$ \\
\hline Mouton $n^{0} 1$ & $\begin{array}{l}\text { Foie } \\
\text { Reins } \\
\text { Cuisse } \\
\text { Rate }\end{array}$ & $\begin{array}{c}12,5 \\
8 \\
2 \\
10,5\end{array}$ & $\begin{array}{c}1,5 \\
1 \\
0 \\
-\end{array}$ \\
\hline Mouton $n^{\circ} 2$ & $\begin{array}{l}\text { Foie } \\
\text { Reins } \\
\text { Gigot }\end{array}$ & $\begin{array}{c}9,2 \\
16 \\
3\end{array}$ & $\begin{array}{c}2 \\
1,5 \\
0\end{array}$ \\
\hline Mouton $n^{\circ} 3$ & $\begin{array}{l}\text { Foie } \\
\text { Reins } \\
\text { Gigot }\end{array}$ & $\begin{array}{l}10,5 \\
12 \\
4,5\end{array}$ & $\begin{array}{l}- \\
-\end{array}$ \\
\hline
\end{tabular}

detraiter les animaux « ̀̀ sec », en plaçant d'une part la dose d'arséniate dans des capsules de gélatine de type « auréomycine » suffisamment robustes pour résister aux chocs, bris ou raclages sur les molaires du mouton lors du traitement, d'autre part, en ne donnant que du foin sec et en suspendant toute distribution d'eau, douze heures avant ef deux heures après l'absorption du médicament. L'essai suivant confirme pleinement cette façon d'opérer, car les pertes imputables à l'action de l'arsenic ont alors cessé.

2) Deuxième temps : Pas de diète ; pas d'eau. L'arséniate d'étain est donné en capsules en une seule fois au moyen d'une pince spéciale. Voir le tableau III.

On remarquera :

a). L'efficacité absolve de l'arséniate d'étain sur Moniezia expansa et sur Moniezia benedeni, quelle que soit la dose envisagée.

b) Stilesia globipunctata n'est détruit que partiellement à $350-400 \mathrm{mg}$ et complètement à $500 \mathrm{mg}$. Le même fait avait déjà été constaté avec l'arséniate de plomb, la situation très caractéristique du parasite, dont le scolex est logé profondément dans un nodule au voisinage du duodénum, le rendant peu sensible à l'action des anthelminthiques.

Cette dose de $500 \mathrm{mg}$ par tête, dans des conditions d'expérience à peu près semblables, correspond exactement à la moitié de celle recommandée dans le traitement du' téniasis ovin avec l'arséniate de plomb. Elle démontre en 
TABLEAU $N^{\circ}$ III

Administration d'arséniate d'étain en capsies, en me fois, sans eau, sans diète préalable

\begin{tabular}{|c|c|c|c|c|c|c|}
\hline $\begin{array}{l}\text { Doses } \\
\text { (par têtc) } \\
\text { en mg. }\end{array}$ & $\begin{array}{c}\text { Nombre } \\
\text { d'animaux }\end{array}$ & Poids（kg） & Parasites en cause & $\begin{array}{l}\text { Efficac } \\
\text { Extensive } \\
\text { E.E. }\end{array}$ & $\begin{array}{l}\text { ité }(\%) \\
\text { Intensive } \\
\text { I.E. }\end{array}$ & Wortalité \\
\hline 250 & $\begin{array}{l}2 \\
1\end{array}$ & ${ }_{20}^{21,20}$ & $\begin{array}{c}\text { Honiezia expansa } \\
\text { Stilesia globipunctata }\end{array}$ & $\begin{array}{r}100 \\
0\end{array}$ & $\begin{array}{r}100 \\
0\end{array}$ & Nulle \\
\hline 350 & $\begin{array}{l}4 \\
1 \\
3\end{array}$ & $\begin{array}{c}26,26,24,22 \\
22 \\
26,26,21\end{array}$ & $\begin{array}{c}\text { Moniezia expensa } \\
\text { Moniezia benederi } \\
\text { Stilesia globizunctata }\end{array}$ & $\begin{array}{r}100 \\
100 \\
33\end{array}$ & $\begin{array}{r}100 \\
100 \\
50\end{array}$ & $\begin{array}{l}n \\
n \\
n\end{array}$ \\
\hline 400 & $\begin{array}{l}1 \\
1 \\
2 \\
2\end{array}$ & $\begin{array}{c}18 \\
23 \\
19,23 \\
19,18\end{array}$ & $\begin{array}{c}\text { Moniezia erpansa } \\
\text { Moniezia benedeni } \\
\text { Stilesia globipunctata } \\
\text { Avitellina centripunctata }\end{array}$ & $\begin{array}{r}100 \\
100 \\
50 \\
100\end{array}$ & $\begin{array}{r}100 \\
100 \\
50 \\
100\end{array}$ & $\begin{array}{l}" 1 \\
" 1 \\
" 1\end{array}$ \\
\hline 500 & $\begin{array}{l}3 \\
3\end{array}$ & $\begin{array}{l}17,18,22 \\
20,18,27\end{array}$ & $\begin{array}{c}\text { Moniezia expansa } \\
\text { Stilesia globipunctata }\end{array}$ & $\begin{array}{l}100 \\
100\end{array}$ & $\begin{array}{l}100 \\
100\end{array}$ & $"$ \\
\hline
\end{tabular}

outre la valeur et l'exactitude de la méthode permettant de tester les ténifuges sur souris (Castel et Gas, 1959).

Ce dosage assure donc la disparition de tous les Moniezia, de Stilesia globipunctata et d'Avitellina centripunctata. Réservée en août 1959 à 10 moutons devant servir ultérieurement à la fabrication de: vaccin antirabique, la dose de $500 \mathrm{mg}$ a été bien supportée, malgré la saison des pluies, l'absence de pâturages, alors inondés, ef la présence d'un parasitisme massif à base d'Haemonchus contortus, d'OEsophagostomum columbianum (adultes et larves) et de paramphistomes divers. Cing autres animaux ont reçu la même quantité de produit le 23-12-1959. Ils sont actuellement en bonne santé ef leur croissance est parfaitement normale.

\section{3) Troisième temps : Diète totale de 20 heures.}

- L'arséniate est distribué en capsules en une seule fois au moyen d'une pince spéciale.

Cependant, vu l'importance attachée par les Russes à cette question (Chubabriya, 1958 ef 1959), une série d'essais supplémentaires a été effectuée en faisant précéder le traitement d'une mise à la diète absolve d'au moins 20 heures.

Les résultats figurent au tableau iV.

Les changements, en ce qui concerne Moniezia expansa. Moniezia benedeni et Stilesia hepatica, sont, dans l'ensemble, peu visibles, Par contre,
Stilesia globipunctato disparaît complètement à des doses $(250 ; 300 ; 350 \mathrm{mg})$ où l'arséniate, sans diète, n'a qu'une action nulle ou limitée (50 p. 100), ainsi que lc montrc la courbe cijointe (Fig. 2). Chubabriya (1958) fait la même constatation avec Moniezia expansa et Avitellina centripunctato : lorsque la durée du jeûne n'est que de 13 à 15 heures, l'efficacité de l'arséniate baisse de 13 p. 100 et après 12 heures, de 20 p. 100.

La diète de 20 heures avant le traitement est donc fortement recommandable, car elle augmente l'activité du corps tout en permettant d'en diminuer la dose qui ne présente alors pratiquement plus aucun risque.

Dans les conditions de cette expérience et dans le but de fixer définitivement la dose la plus appropriée, nous avons dọ tenir compte de plusieurs données :

- Le trop petit nombre de moutons touchés par Stilesia globipunctata, soumis aux doses de 250-300 mg par tête.

- L'irrégularité d'action des doses supérieures (90 p. 100 d'efficacité à $400 \mathrm{mg}$ ).

- Les excellents résultats obtenus, toujours avec Stilesia globipunctata, sur les huit moutons traités avec $350 \mathrm{mg}$ d'arséniate.

- Les 86 p.'100 d'l. E. du produit sur Avitellino centripunctata à la dose de $300 \mathrm{mg}$.

- Les 100 p. 100 d'efficacité sur Moniezia expansa el sur Moniezia benedeni. Aussi avons- 
TABLEAU $\because 0$ Ï

Admiristration d'arséniate d'étain en capsules, en une fois, après une diète totale de 20 heures

\begin{tabular}{|c|c|c|c|c|c|c|}
\hline $\begin{array}{l}\text { Doses } \\
\text { (par tête) } \\
\text { en mg. }\end{array}$ & $\begin{array}{l}\text { liombre } \\
\text { d'animaux }\end{array}$ & Poids (kg) & Parasites en cause & $\begin{array}{l}\text { Efficac } \\
\text { Extensive } \\
\text { E.E. }\end{array}$ & $\begin{array}{l}\text { té }(\%) \\
\text { Intensive } \\
\text { I.E. }\end{array}$ & Mortalité \\
\hline 250 & $\begin{array}{l}4 \\
1 \\
2 \\
2\end{array}$ & $\begin{array}{c}20,19,20,18 \\
22 \\
21,20 \\
20,20\end{array}$ & $\begin{array}{c}\text { Moniezia expensa } \\
\text { Moniezia benedeni } \\
\text { Stilesia globipunctata } \\
\text { avitellina centripunctata }\end{array}$ & $\begin{array}{l}100 \\
100 \\
100 \\
100\end{array}$ & $\begin{array}{l}100 \\
100 \\
100 \\
100\end{array}$ & $\begin{array}{c}\text { Nulle } \\
" \\
" \\
"\end{array}$ \\
\hline 300 & $\begin{array}{l}1 \\
2 \\
1\end{array}$ & 20,24 & $\begin{array}{l}\text { Stilesia globipunctata } \\
\text { Avitellina centri punctata } \\
\text { Stilesia hepatica }\end{array}$ & $\begin{array}{r}100 \\
50 \\
0\end{array}$ & $\begin{array}{r}100 \\
86 \\
0\end{array}$ & $\begin{array}{l}" \\
" 1\end{array}$ \\
\hline 350 & $\begin{array}{l}3 \\
1 \\
8 \\
2 \\
1 \\
1\end{array}$ & $\left\{\begin{array}{c}21,33,28 \\
27 \\
20,21,22,18 \\
25,28,21,23 \\
23,32 \\
27 \\
21\end{array}\right.$ & $\begin{array}{l}\text { Moniezia expansa } \\
\text { Iloniezia benedeni } \\
\text { Stilesia globipunctata } \\
\text { Stilesia hepatica } \\
\text { Avitellina centripunctata } \\
\text { Avitellina woodlandi }\end{array}$ & $\begin{array}{r}100 \\
100 \\
100 \\
0 \\
100 \\
100\end{array}$ & $\begin{array}{r}100 \\
100 \\
100 \\
0 \\
100 \\
100\end{array}$ & $\begin{array}{l}" \\
" \\
" \\
" \\
"\end{array}$ \\
\hline 400 & $\begin{array}{l}2 \\
4 \\
2 \\
2\end{array}$ & $\begin{array}{c}27,25 \\
20,22,25,28 \\
32,22 \\
25,27\end{array}$ & $\begin{array}{l}\text { Honiezia expansa } \\
\text { Stilesia globipunctata } \\
\text { Stilesia hepatica } \\
\text { Avitellina centripunctata }\end{array}$ & $\begin{array}{r}100 \\
75 \\
0 \\
100\end{array}$ & $\begin{array}{r}100 \\
90 \\
0 \\
100\end{array}$ & " \\
\hline 500 & $\begin{array}{l}2 \\
1\end{array}$ & $\underset{25}{21,25}$ & $\begin{array}{l}\text { Moniezia expansa } \\
\text { Stilesia globipunctata }\end{array}$ & $\begin{array}{l}100 \\
100\end{array}$ & $\begin{array}{l}100 \\
100\end{array}$ & $"$ \\
\hline
\end{tabular}

nous choisi un chiffre moyen et la dose de $350 \mathrm{mg}$ par animal en une seulc fois a ćté jugéc la plus satisfaisante d̀ tous égards, puisqu'elle détruit tous les Moniezia, tous les Avitellina et à près tous les Stilesia. Les doses plus faibles, bien que très bonnes, ne semblent pas d'une sécurité absolue et des échecs sont possibles, les doses plus fortes ne paraissent pas plus efficaces et il est inutile d'accroître les quantités d'arséniate à administrer si les résultats ne doivent pas être meilleurs.

\section{4) Conclusions.}

a) La dose de $250 \mathrm{mg}$ par tête, en une seule fois et en capsules rigoureusement dosées, convient parfaitement bien à tous les moutons hébergeant Moniezio expanso. Elle sera administrée « à sec » avec ou sans diète de 20 heures, quoique cette dernière soit nettement préférable.

Elle est totalement inoffensive et ne fait courir aucun risque ni à l'animal ni au consommateur. Les Moniezia expansa étant les Anoplocephalinae les plus répandus en Europe, il serait souhai- table que l'arséniate d'étain trouve une large audience dans les milieux s'occupant de la prophylaxie du téniasis ovin dans cette partie du monde.

b) La dose de $350 \mathrm{mg}$, en capsules et après une diète absolve de 20 heures, sera préconisée de préférence dans les pays où se manifestent plusieurs espèces d'Anoplocephalidae à la fois, seules ou associées. C'est le cas de beaucoup de pays tropicaux. La dose suffit à assurer, sans incidents notables la destruction de la quasitotalité des cestodes du mouton. L'époque des traitements sera fixée dans chaque zone en fonction des conditions épidémiologiques propres au téniasis ovin.

c) La dose de $500 \mathrm{mg}$ en capsules, « à sec 》, sans diète, et en une seule fois, entraîne, dans tous les cas, l'expulsion de Moniezio benedeni, Moniezia exponsa. Stilesia globipunctata, Avitellina centripunctoto et Avitellina woodlandi. Stilesio hepatica demeure indemne. Quant à Helictometra ovilla, il n'a pu faire l'objet d'investigations faute de matériel. 
FIQ. 2. POURCENTAGE Dי EPPICACI TB SUR GTILESIA QLOBIPUNCTATA

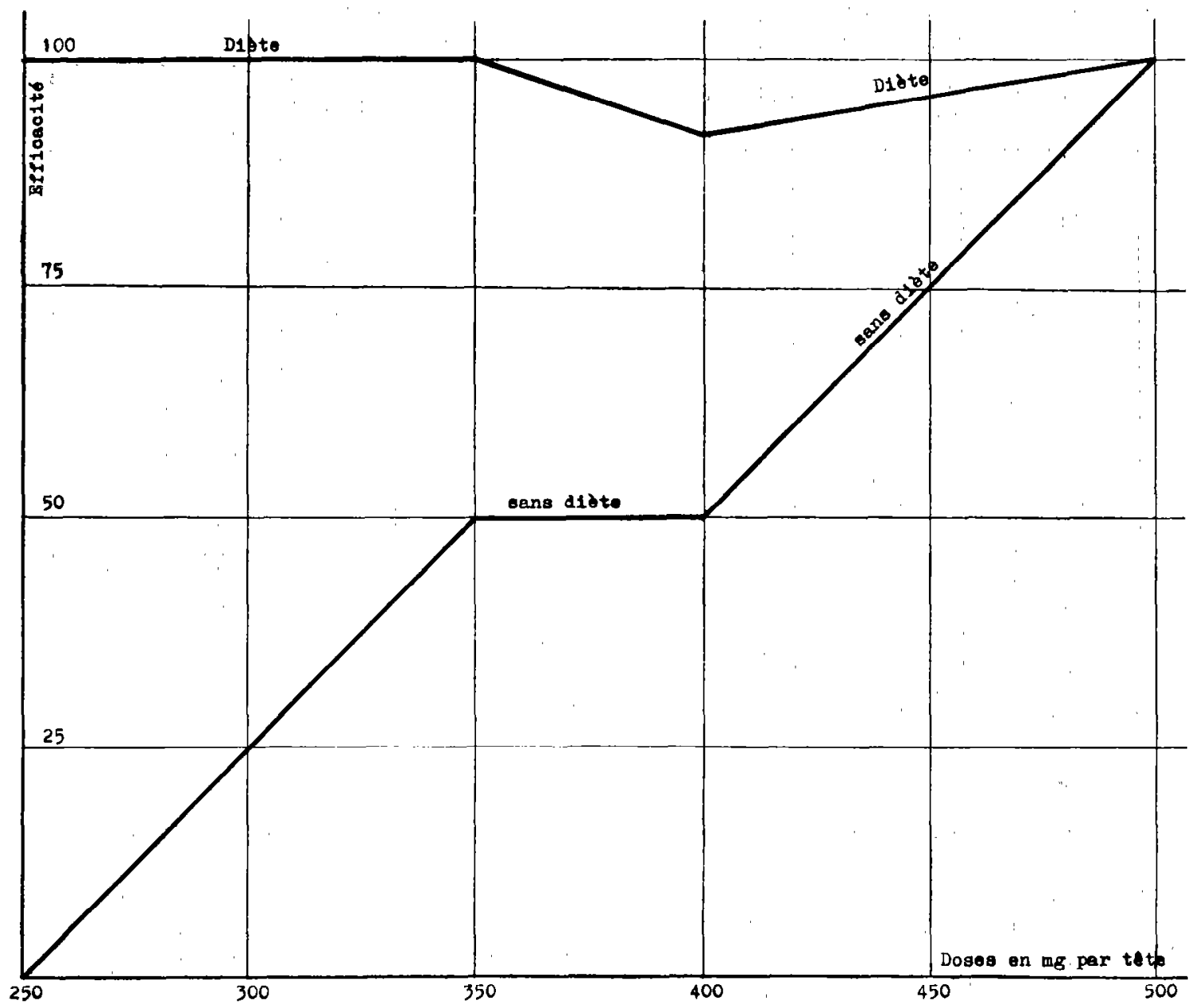

Cette dose paraît très séduisante puisqu'elle réduit au minimum le temps de préparation ef d'immobilisation de l'animal. Etant déjà assez forte, elle demande néanmoins à être manipulée avec prudence, en tenant compte de la saison, de l'état des animaux, du parasitisme associé et de la rickettsiose, ainsi qu'il sera dit plus loin.

Ces résultats diffèrent sensiblement de ceux indiqués par les chercheurs russes placés dans les mêmes conditions que nous, à savoir : diète de 18-20 heures avant le traitement ef mise en capsules de l'arséniate. Le tableau Ven témoigne.

N'ayant pu comparer les deux produits, il est difficile d'émettre un jugement. Tout ce que l'on peut dire, c'est que notre arséniate semble se différencier de l'arséniate russe par l'absence d'une demi-molécule d'eau et qu'il a été préparé à partir de corps très purs donnant toutes garanties quant à l'utilisation d'un produit défini.

\section{E) MODE D'ACTION}

La mort des Moniezia est brutale. Ils se segmentent en de multiples fragments plus ou moins longs, avant de passer à l'extérieur. II n'est pas rare de rencontrer des scolex. II n'existe plus d'anneaux dans les excréments 72-85 heures après le traitement.

Les Avifellina sont rapidement touchés : au bout de 24 heures, il n'en reste plus aucun en vie. L'évacuation est terminée en 72 heures. Généralement, le cestode est expulsé entier, déjà en partie digéré par les sucs digestifs intestinaux, ce qui lui donne une couleur jaunebistre ef un aspect « ratatiné » caractéristique. 


\section{TABLEAU $\mathrm{H}^{\circ} \mathrm{V}$}

Adninistration au mouton d'arséniate d'étain en apsules après une diète totale de 20 heures. Résultats en U.R.S.S. et au Tahad

\begin{tabular}{|c|c|c|c|}
\hline Parasites & Doses en $g$ & Auteurs & Tchad \\
\hline $\begin{array}{l}\text { Moniezia expanse } \\
\text { Moniezia benedeni }\end{array}$ & $\begin{array}{c}0,5 \\
0,3-0,4 \\
1-4 \text { mois: } 0,4 \\
4-6 \text { mois: } 0,6 \\
6-8 \text { mois: } 0,8\end{array}$ & $\begin{array}{l}\text { Garkavi, } 1956 \\
\text { Chubabriya, } 1958 \\
\left\{\begin{array}{rr}1957 \\
\text { et } \\
1959\end{array}\right.\end{array}$ & $\begin{array}{l}\text { Dose uniforme } \\
\text { de } 0,25 \mathrm{~g} \\
\text { pour des anjmaux de } \\
14 \text { a } 32 \mathrm{~kg}\end{array}$ \\
\hline $\begin{array}{l}\text { Avitelling } \\
\text { centripunctata }\end{array}$ & $\begin{array}{c}1 \text { par tête } \\
0,7-1\end{array}$ & $\begin{array}{l}\text { Grigoryan, } 1957 \\
\text { Ulyanov, } 1957\end{array}$ & $\begin{array}{l}\text { 0,35 \& par tete, } \\
\text { quel que soit le poids }\end{array}$ \\
\hline $\begin{array}{l}\text { Stilesia } \\
\text { Globipunctata }\end{array}$ & \multicolumn{2}{|c|}{ Pas de travaux russes } & 0,35 par tête \\
\hline Stilesia hepatios & \multicolumn{2}{|c|}{ Pas de travaux russes } & $\begin{array}{l}\text { Inefficece, } \\
\text { quelle que soit la dose }\end{array}$ \\
\hline $\begin{array}{l}\text { Helictometra } \\
\text { ovilla }\end{array}$ & $\begin{array}{l}0,7-1 \\
0,7=1\end{array}$ & $\begin{array}{c}\text { Ulyanov, } 1957 \\
\text { Chubabriys, } 1959\end{array}$ & Pas de travaux français \\
\hline
\end{tabular}

Les Stilesia mettent plus longtemps à disparaître : de 24 à 48 heures. Ils adhèrent encore quelques hcures à la muqueuse intestinale avant de s'en détacher, pour ne laisser subsister que des nodules bien visibles, mais vides de scolex. On ne voit pratiquement pas d'anneaux de Stilesia dans les crottes. Cet Anoplocephalidae très fin et très délicat paraît totalement digéré au cours du transit dans l'intestin de son hôte.

L'arséniate d'étain agit donc avec célérité en tuant, selon un processus que nous ignorons, les Moniezia, les Stilesia et les Avitellina qui sont éliminés au plus tard dans les 96 heures qui suivent l'administration du produit.

\section{F) CONSÉQUENCES DU TRAITEMENT A L'ARSÉNIATE D'ÉTAIN}

\section{I. - Sur le mouton}

Elles sont faibles. L'arséniate ne détermine que peu de changements dans l'attitude et le comportement des animaux traités. Tout au plus relève-t-on des manifestations de tristesse ef de l'inappétence, surtout aux doses les plus fortes (400 et $500 \mathrm{mg}$ ). Quelques cas de diarrhée ont été observés. lls ne durent pas et au bout de 48 heures, les perturbations cessent.

\section{II. - Sur les brebis pleines}

Le traitement est parfaitement toléré, à condition d'être effectué un bon mois avant l'époque de la mise-bas. Les quatre femelles droguées (deux à $250 \mathrm{mg}$ et deux à $350 \mathrm{mg}$ ) ont normalement conduit leur gestation et ont mis au monde des agneaux bien vivants.

\section{III. - Sur les éléments du sang}

Ils ne subissent pas dans l'ensemble de modifications sensibles immédiatement; après l'administration d'arséniate. Chez un certain nombre de moutons, tant à la dose de $250 \mathrm{mg}$ qu'à celle de $350 \mathrm{mg}$, on note, deux jours après le traitement, une diminution plus ou moins accusée du nombre d'hématies et de neutrophiles, ainsi qu'une augmentation du nombre de lymphocytes. On sait qu'avec l'arsenic à des doses répétées pendant un laps de temps assez long, il en est quelquefois ainsi (Talbert et Tayloe, 1933). Dans le cas qui nous intéresse, ces variations n'ont pas de signification et, 15 jours après, tout est rentré dans l'ordre. Sur la moitié des ani- 
maux, le nombre d'hématies est même supérieur : à ce qu'il était au départ (de 10 à 22 p. 100 en plus).

\section{IV. - Augmentation de poids}

Elle est spectaculaire. L'essai a porté sur douze moutons, répartis en trois lots égaux:

Dans le premier lot, chaque mouton a reçu $250 \mathrm{mg}$ d'arséniate.

Dans le deuxième lot, chaque mouton a reçu $350 \mathrm{mg}$ d'arséniate.

Le troisième lot comprenait des témoins tous parasités. Ces animaux ont été mis au pâturage pendant deux mois (tableau VI).

\section{G) MODE D'ADMINISTRATION}

\section{1. - Préparation de l'animal}

o) La diète absolve est strictement nécessaire dans le traitement de la stilésiose et de l'avitellinose ovines $(350 \mathrm{mg})$, facultative, mais fortement recommandable lors de moniéziose (250 mg). Nous opérons généralement cinsi : l'animal est laissé à jeun la veille vers 12 heures dans un local cimenté, sans paille et sans eau. II n'est traité que le lendemain vers 8 heures. De l'eau en petite quantité et' du foin bien sec à satiété ne lui seront redonnés que vers 9 heures et

TABLEAU No $\mathrm{I}$

Augmentation du poids des moutons traités à l'arséniate d'étain

\begin{tabular}{|c|c|c|c|c|c|}
\hline $\begin{array}{c}\text { Doses } \\
(\mathrm{mg})\end{array}$ & $\begin{array}{c}\text { Poidde moyen } \\
\text { au début } \\
(\mathrm{kg})\end{array}$ & $\begin{array}{c}\text { Un mois après } \\
(\mathrm{kg})\end{array}$ & $\begin{array}{c}2 \text { mois après } \\
(\mathrm{kg})\end{array}$ & \multicolumn{2}{|c|}{ Augmentation } \\
\cline { 4 - 6 } & 22,750 & 27,250 & 28 & 5,250 & 23,4 \\
\hline 250 & 22,750 & 24,500 & 27,250 & 4,500 & 19,7 \\
350 & 24,450 & 27,750 & 28,250 & 3,800 & 15,3 \\
\hline
\end{tabular}

L'augmentation de poids est de $5,250 \mathrm{~kg}$ à $250 \mathrm{mg}$ et de $4,500 \mathrm{~kg}$ d̀ $350 \mathrm{mg}$. Elle est légèrement supérieure à celle mentionnée par les Russes en Géorgie (Chubabriya, 1959). Ce dernier cite le chiffre de $3,800 \mathrm{~kg}$ par animal, sans préciser la durée de l'expérience. Sur la toison, le gain est de $350 \mathrm{gr}$. L'arséniate d'étain, grâce à l'arsenic' renfermé d'une part, et à la disparition des cestodes d'autre part, provoque un véritable '«coup de fouet» particulièrement. évident à la dose de $250 \mathrm{mg}$ : 19 p. 100 d'augmentation de poids en un mois contre 7,7 p. 100 à $350 \mathrm{mg}$ et 12,1 p. 100 pour les témoins. L'arsenic, d'après certains travaux faits sur le lapin (Sivadjian, 1946), amènerait, sans accroissement notable de la quantité d'aliments apportés, une meilleure utilisation de ceux-ci: Les effets du « coup de fouet » arsenical sont de courte durée et ils s'estompent dès le second mois, Ils suffisent néanmoins à remettre en état, dans un temps record, un troupeau de moutons fortement infestés. demi-10 heures. II est remis au: pâturage vers 12 heures.

L'essentiel est d'éviter une absorption massive d'eau aussitôt après le traitement', sinon 'une trop grande quantité d' $\mathrm{As}_{2} \mathrm{O}_{5}$ risque de se former et d'aller intoxiquer l'animal. C'est ce qui a eu lieu deux fois, à la dose de $500 \mathrm{mg}$, les moutons ayant bu quinze minutes après le traitement.

Dans les pays où les ovins pâturent des herbes vertes chargées d'eau (pays tempérés ; zone tropicale pendant l'hivernage), on a intérêt, pour plus de sécurité, à reculer l'époque où la diète doit prendre fin. Ce délai sera alors de quatre heures au minimum. La marche à suivre peut être résumée ainsi :

$\mathrm{Ni}$ eau, ni foin : de 12 heures la veilleà 8 heures le lendemain.

Traitement : à 8 heures du matin.

Ni foin, ni equ: de 8 heures à 12 heures.

Foin à volonté. - Pas d'herbes vertes. - Un peu d'eau : à 12 heures.

Remise au pâturage : vers 15 heures. 
Cet horaire demande à être scrupuleusement respecté.

b) Lors de moniéziose ovine d̀ Moniezia expansa $(250 \mathrm{mg}$ ), il est possible de ne pas soumettre l'animal à un régime aussi sévère. On traite « à sec », c'est-à-dire en supprimant toufe distribution d'eau pendant au moins 18 heures.

On procède généralement de cette façon :

Mise en bergerie des moutons vers 18 heures le soir avec du foin sec sans eau.

Traitement le lendemain vers 7 heures.

Foin, sans eau, de 7 heures à 11 heures.

Abreuvement et envoi des animaux au pâturage à partir de 12 heures.

La technique est valable également pour la dose de $500 \mathrm{mg}$ par tête.

\section{II. - Administration du produit.}

Comme nous l'avons déjà écrit, l'arséniate est administré en capsules de gélatine rigoureusement dosées.

Il existe de nombreux moyens de faire pénétrer la capsule dans l'œsophage du mouton. Le plus simple consiste à utiliser une pince spéciale dite «pince lance-capsule » (fig. 3) munie

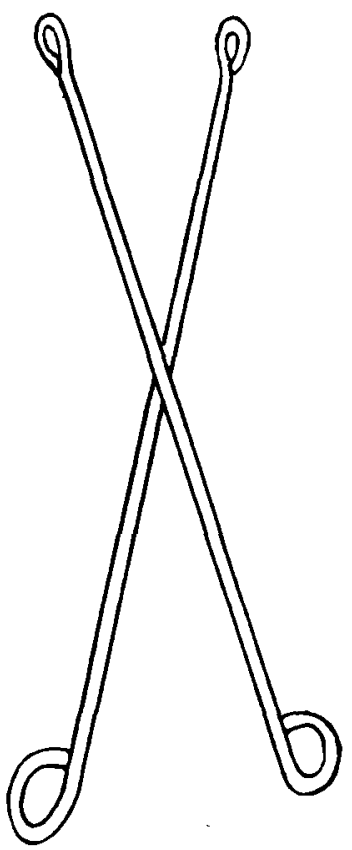

Fig. 3 - Pince lance-capgrulo à l'une de ses extrémités de deux oreilles entre lesquelles on coince la capsule. Un aide écarte largement les deux branches du maxillaire de l'animal. La pince est glissée au fond de la gorge et la capsule libérée.

Les Russes (Chubabriya, 1959) ont mis au point (fig. 4) un pistolet capable d'emmaganiser 20 à 30 capsules à la fois, selon leur taille. Un système de ressort permet leur expulsion une par une. Le canon est introduit jusqu'à la base de la langue.

Le système est pratique, car les capsules sont totalement protégées des raclages et des bris sur les dernières molaires. De plus, elles parviennent sous une certaine pression dans l'arrière-gorge et ont, de ce fait, plus de chances d'être avalées sans incident par l'animal.

\section{H) TOXICITÉ}

Nous envisagerons :

1) La foxicité de l'arséniate d'étain pour le mouton.

2) La toxicité pour l'homme de la viande et des organes des animaux traités.

\section{1) Toxicité de l'arséniate d'étain pour le mouton}

\section{a) Doses toxiques}

D'après les travaux de 1. S. Mosgov (Chubabriya, 1958). la dose de $2,5 \mathrm{~g}$ d'arséniate est tenve pour dose toxique non mortelle (mouton de $30 \mathrm{~kg}$ ). Pour Chubabriya (1958), la dose mortelle est de $3 \mathrm{~g}$. Chez les agneaux de 10 à $28 \mathrm{~kg}$, deux grammes constituent une dose toxique non

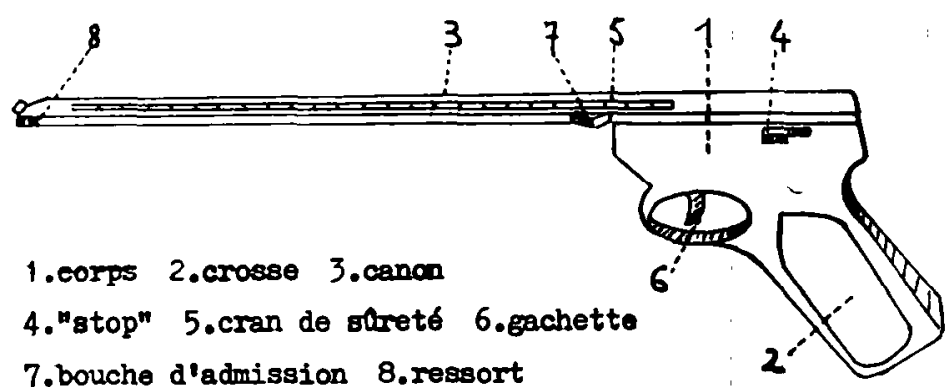

F18.4 - P1stolet lanco-aspoule (d'aprib Chubabriya, 1959) 
mortelle, tandis qu'une dose de $1,5 \mathrm{~g}$ n'est pas toxique (Chubabriya, 1959). Gelovani (1958) et Mamatelashvili (1958) considèrent que des doses voisines de $50 \mathrm{mg} / \mathrm{kg}$ ne sont pas toxiques pour des moutons de 3 à 4 mois. La dose létale est comprise entre 110 et $130 \mathrm{mg} / \mathrm{kg}$.

Cette question a été reprise tant au Laboratoire de Farcha qu'au Laboratoire de Pharmacie chimique de la Faculté de Montpellier :

Doses de $2,5 \mathrm{~g}$ (Fort-Lamy) : les deux moutons traités (29 et $30 \mathrm{~kg}$ ) après diète de 20 heures, sont morts 36 et 48 heures après l'administration du produit.

Doses de 2,25 g (Fort-Lamy) : les deux moutons $(27$ et $31 \mathrm{~kg}$ ) meurent 24 et 48 heures après le traitement.

Doses de $2 \mathrm{~g}$ (Fort-Lamy) : un mouton de $23 \mathrm{~kg}$. Cet animal a survécu 47 jours avant d'être sccrifié.

Doses de 1,8 g (Montpellier) : Quatre brebis pesant de 20 d̀ $25 \mathrm{~kg}$ ont reçu $1,8 \mathrm{~g}$ d'arséniate d'étain en capsules, après une diète de 20 heures. Elles n'ont manifesté aucun signe d'intoxication. Deux d'entre elles ont subi l'examen anatomopathologique: Il n'a pas été rencontré de lésions histologiques caractéristiques.

Doses de $1 \mathrm{~g}$ (Fort-Lamy) : l'animal (27 kg) a très bien résisté. 'II n'est mort qu'au bout de 45 jours d'une schistosomiase.

De tous ces essais, il résulte qu'avec l'arséniate utilisé, la dose mortelle pour le mouton se silue aulour de $2,25 \mathrm{~g}$. Pour des animaux de même poids, notre produit est donc plus toxique que le produit russe (dose létale : 2,25 g contre $3 \mathrm{~g}$ ). Cette différence, surprenante au premier abord, pourrait être expliquée par la résistance des ovins aux anthelminthiques, résistance qui varie selon les pays, les races ef les conditions d'entretien.

L'écart entre la dose thérapeutique et la dose mortelle est de 8 à $9(250 \mathrm{mg})$ de $6(350 \mathrm{mg})$ et de 4 (500 mg). Il s'agit là d'un excellent résullat : l'arséniate d'étain laisse une grande marge de sécurité, surtout aux doses les plus faibles (250 et $350 \mathrm{mg}$ ).

Les symptômes et les lésions de l'intoxication arsenicale, tels qu'ils s'extériorisent au Tchad, ont été décrits plus haut. A la dose de $2,5 \mathrm{~g}$, les signes s'exacerbent : coliques violentes : l'animal se couche et se relève constamment, les naseaux dilatés, la respiration haletante ; soif intense et diarrhée.

A l'autopsie, les lésions les plus apparentes se voient au niveau de l'intestin, siège d'une inflammation aiguë avec plaques hémorragiques par endroit. L'organe est rempli de sang et ses parois deviennent extrêmement minces. II en est de même pour la caillette. On note également des lésions de néphrite et le foie prend une couleur feuille morte. Ce tableau correspond d̀ celui qui est classiquement décrit lors d'intoxication arsenicale (Adams ef Hugues, 1955).

\section{b) Influences des conditions extérieures locales sur la toxicité de l'arséniate d'étain}

En milieu tropical, il importe de tenir compte des facteurs ambiants, de manière à éviter les accidents susceptibles de se produire après l'administration d'arséniate d'étain à des doses déjà fortes $(500 \mathrm{mg})$.

La période de l'année favorable à l'élevage du mouton semble très courte dans les régions sahéliennes du Tchad : elle ne dépasse pas 5 mois (du 15 novembre au 15 avril). Passé ce temps, l'animal est soumis à l'action de causes diverses telles qu'alimentation et abreuvement insuffisants en quantité comme en qualité, tornades, helminthiases variées, seules ou associées (Haemonchus et œesophagostomes), tiques dont Amblyomma variegatum, vecteur de Cowdria ruminantium agent de la heart-water. Tous ces facteurs amoindrissent sensiblement la résistance de l'animal à l'action des Anoplocephalidue d'une part et à celle des ténifuges employés d'autre part.

1) Pratiquement, il est toute une période de l'année pendant laquelle il ne faut pas songer à déparasiter les moutons sous peine de les voir succomber de heart-water dans 80 p. 100 des cas environ. Les rickettsies se mobilisent à la suite du traitement à l'arséniate d'étain et l'animal meurt avec des signes qui ne sont pas ceux d'une intoxication arsenicale. Le même fait a été observé à la même époque sur des bouvillons et des moutons ayant reçu des anthelminthiques d'ordinaire anodins, tels que de la phénothiazine ou divers dérivés de la pipérazine.

Cette période va, dans les conditions de FortLamy, du 15 août au 15 novembre. Des essais effectués en septembre 1959 sur 7 moutons, à la dose de $500 \mathrm{mg}$, en apportent la preuve : cinq 
d'entre eux ont succombé des suites d'une rickettsiose aiguë ou suraiguë (avec mise en évidence des parasites dans les vaisseaux du cerveau) dans les deux jours faisant suite au traitement. Les deux autres moutons non atteints, ont servi à d'autres expériences un mois plus tard. Bien entendu, il s'agit de conditions propres à la zone de Fort-Lamy; il n'est pour l'instant pas question, faute de renseignements supplémentaires, d'étendre ces constatations à l'ensemble du pays. Cependant, toutes les fois qu'il s'avérera nécessaire de traiter des moutons contre le téniasis à l'époque indiquée, l'opérateur devra procéder avec prudence et il serait bon de «tâter le terrain » sur un petit nombre d'animaux, avant de songer à déparasiter l'ensemble.

2) Les ovins en très mauvais état, maigres, insuffisamment nourris ou trop anémiés, sont capables de mal supporter le traitement. Nous avons eu quelques incidents de cette nature, début août 1959 (à $500 \mathrm{mg}$ ), avec diarrhée, amaigrissement rapide et mort 7 à 8 jours plus tard.

Aussi, de mai à novembre, faudra-t-il, dans les zones tropicales subsahariennes, faire très attention et s'entourer de toutes les garanties possibles : diète absolve de 20 hcures avant et de 4 heures après la distribution du ténifuge. Pas d'eau. Foin sec : pas d'herbes vertes. Les animaux souffreteux ou malades seront isolés et ne recevront au départ qu'une faible dose (250 mg) qui sera renouvelée quelques jours après. Ces précautions sont surtout valables pour la dose de $500 \mathrm{mg}$ et, dans une moindre mesure, pour $350 \mathrm{mg}$. Par contre, de début novembre à la mi-avril, selon l'année et l'état des pâturages, l'arséniate d'étain, quelle que soit la dose, ne fait courir aucun risque à l'animal. Il permet de lutter efficacement contre le téniasis latent (Euzéby, 1957 ; Graber, 1959) qui représente une grosse menace en fin de saison sèche où les conditions d'entretien du mouton sont telles qu'une ruplure d'équilibre peut se réaliser au profit du ou des cestodes. Le téniasis a alors tendance à passer du stade latent au stade maladie avec toutes les conséquences que cefte helminthiase comporte.

La prophylaxie de masse du téniasis ovin au cours de l'hiver a d'autant plus de chances d'aboutir à un résultat sérieux que les chances d'infestation ou de réinfestation paraissent, d'après les premières observations*, diminuer considérablement au fur et à mesure que la saison sèche s'avance (de janvier à fin mai).

\section{2) Toxicité pour l'homme de la viande de mouton traité à l'arséniate d'éłain}

Deux éléments doivent être pris en considération : l'étain ef l'arsenic.

\section{a) Etain}

Il ne présente pas d'inconvénients. A 350$400 \mathrm{mg}$, les concentrations de ce métal dans la viande des animaux traités sont de 50 à 100 fois plus faibles que celles autorisées dans les conserves.

\section{b) Arsenic}

La législation française interdit, dans les aliments, toute quantité d'arsenic supérieure à $0,1 \mathrm{mg} / \mathrm{kg}$. Des dosages (méthode de Cribier) ont été pratiqués sur des ovins ayant reçu une dose d'arséniate d'étain de $400 \mathrm{mg}$, ou $350 \mathrm{mg}$ ou $250 \mathrm{mg}$. Pour chacune de ces doses on a utilisé deux lots de trois animaux; ceux du premier lot ont été sacrifiés 6 jours après l'administration de l'arséniate ef ceux du deuxième lot 12 jours après (Tableau VII).

A la cuose de $400 \mathrm{mg}$, les dosages effectués sur des moutons tués le sixième jour après le traitement montrent que le rein, la rate, le poumon ef l'intestin renferment encore un peu trop d'arsenic ; par contre, le cœur, le cerveau, la langue ef le foie sont consommables. A $350 \mathrm{mg}$, seuls la rate et l'intestin dépassent le chiffre de $0,1 \mathrm{mg} / \mathrm{kg}$. A $250 \mathrm{mg}$. les organes ne contiennent plus d'arsenic, sauf la rate.

Dans les trois cas, les muscles, c'est-à-dire les gigots, les épaules et les filets sont absolument dépourvus de toute trace d'arsenic.

Douze jours après, que ce soit à 250,350 ou $400 \mathrm{mg}$. l'arsenic a complètement disparu, sauf de la rate, mais il demeure bien en deçà de ce qui est toléré par le législateur.

Ces résultats corroborent ceux obtenus par Nanobashvili (1959) en Géorgie. Nous ferons nôtre sa conclusion : «dans les muscles de l'agneau, après le traitement antiparasitaire à

(*) M. Graber, Résultats non encore publies. 
TABLEAU $\mathrm{H}^{\circ}$ VII

Quantitéa d'arsenic dans différents organes de moutons ayant été traités avec l'arséniate d'étain

\begin{tabular}{|c|c|c|c|c|c|c|c|c|c|}
\hline \multirow{2}{*}{ Doses } & \multirow{2}{*}{ Organes } & \multicolumn{4}{|c|}{6 jourg après en As $\mathrm{mg} / \mathrm{kg}$} & \multicolumn{4}{|c|}{12 jours après en $\mathrm{As} \mathrm{mE} / \mathrm{kg}$} \\
\hline & & $\mathbb{N}^{0} 66$ & No 84 & Hי 171 & Moyenne & No 113 & $N^{\circ} 117$ & No 125 & Moyenne \\
\hline \multirow{11}{*}{$400 \mathrm{mg}$} & Foie , & 0 & 0 & 0,228 & 0,076 & 0 & 0 & 0 & 0 \\
\hline & Rein & 0,185 & 0,202 & 0 & 0,129 & 0 & 0 & 0 & 0 \\
\hline & Rate & 0,807 & 1,262 & 0,553 & 0,874 & 0 & 0,052 & 0,052 & 0,035 \\
\hline & Coeur & 0 & 0 & 0 & 0 & 0 & 0 & 0 & 0 \\
\hline & Poumon & 0 & 0,401 & 0 & 0,133 & 0 & 0 & 0 & 0 \\
\hline & Cerveau & 0 & 0 & 0 & 0 & 0 & 0 & 0 & 0 \\
\hline & Langue & 0 & 0,121 & Traces & 0,040 & 0 & 0 & 0 & 0 \\
\hline & Intestin & - & 0 & 0,406 & 0,136 & - & 0 & 0 & 0 \\
\hline & Gigot & 0 & 0 & 0 & 0 & 0 & 0 & 0 & 0 \\
\hline & Epaule & - & 0 & 0 & 0 & 0 & 0 & 0 & 0 \\
\hline & Filet & & & & & 0 & 0 & - & 0 \\
\hline \multirow{2}{*}{ Doses } & \multirow{2}{*}{ Organes } & \multicolumn{4}{|c|}{7 jours après en As $\mathrm{mg} / \mathrm{kg}$} & \multicolumn{4}{|c|}{12 jours après en As mg $/ \mathrm{kg}$} \\
\hline & & $N^{\circ} 20$ & No 48 & No 50 & Moyenne & $N^{\circ} 145$ & N10 146 & 칭 147 & Hoyenne \\
\hline \multirow{11}{*}{$350 \mathrm{mg}$} & Foie & 0 & 0 & traces & 0 & 0 & 0 & 0 & 0 \\
\hline & Rein & 0 & 0,051 & 0,030 & 0,040 & 0 & 0 & 0 & 0 \\
\hline & Rate & 0,204 & 0,322 & 0,182 & 0,236 & 0,032 & 0,021 & $0 ; 040$ & 0,031 \\
\hline & Coeur & - & 0 & 0 & 0 & 0 & 0 & 0 & 0 \\
\hline & Poumon & - & 0 & 0 & 0 & 0 & 0 & 0 & 0 \\
\hline & Cerveau & 0 & 0 & 0 & 0 & 0 & 0 & 0 & 0 \\
\hline & Langue & - & - & - & - & 0 & 0 & 0 & 0 \\
\hline & Intestin & 0,082 & 0,122 & 0,201 & 0,135 & 0 & 0 & 0 & 0 \\
\hline & Gigot & 0 & 0 & 0 & 0 & 0 & 0 & 0 & 0 \\
\hline & Filet & 0 & 0 & 0 & 0 & 0 & 0 & 0 & 0 \\
\hline & Epaule & 0 & 0 & 0 & c & 0 & 0 & $0^{\prime}$ & $0^{\prime}$ \\
\hline \multirow{2}{*}{ Doses } & \multirow{2}{*}{ Organes } & \multicolumn{4}{|c|}{6 jours après en $\mathrm{As} \mathrm{mg} / \mathrm{kg}$} & \multicolumn{4}{|c|}{12 jours après en $\mathrm{As} \mathrm{mg} / \mathrm{kg}$} \\
\hline & & $\mathbb{N}^{\circ} 148$ & $\mathbb{N}^{0} 149$ & $N^{0} 150$ & Moyenne & No 151 & $\mathbb{N}^{0} 952$ & $N^{0} 153$ & Moyenne \\
\hline \multirow{11}{*}{$250 \mathrm{mg}$} & Foie & 0 & 0 & 0 & 0 & & 0 & 0 & 0 \\
\hline & Rein & 0 & 0 & 0 & 0 & & 0 & 0 & 0 \\
\hline & Rate & 0 & 0,052 & 0,052 & 0,035 & & .0 & traces & \\
\hline & Coeur & 0 & 0 & 0 & 0 & & 0. & 0 & 0 \\
\hline & Poumon & 0 & 0 & 0 & 0 & & 0 & 0 & 0 \\
\hline & Cervean & 0 & 0 & - & 0 & . & 0 & 0 & $: 0$ \\
\hline & Iangue & 0 & 0 & - & 0 & & 0 & 0 & 0 \\
\hline & Intestin & - & 0 & 0 & 0 & & 0 & 0 & 0 \\
\hline & Gigot & 0 & 0 & 0 & 0. & - & 0 & 0 & 0 \\
\hline & Filet & 0 & 0 & 0 & 0 & & 0 & 0 & 0 \\
\hline & Epaule & 0 & 0 & 1 & 0 & & & $0:$ & 0 \\
\hline
\end{tabular}


l'arséniate d'étain à la dose de 0,3-0,4 g par tête, l'arsenic, en quantité mesurable, se décèle seulement le jour où l'on administre la préparation. Dès le second jour et les 4 ou 5 jours suivants, on ne peut retrouver dans les muscles de l'animal que des traces d'arsenic. II en résulte que la viande de mouton peut être vendue pour la consommation humaine 4 ou 5 jours après le traitement antiparasitaire $\gg$.

C'est ainsi qu'à Fort-Lamy, des moutons qui avaient été traités à l'arséniate d'étain, deux avec $500 \mathrm{mg}$, neuf avec $350 \mathrm{mg}$ et neuf avec $250 \mathrm{mg}$, ont été débités et mangés à notre insu, 6,7 ef 12 jours respectivement après la fin $d u$ traitement, par les mancuvres du laboratoire qui n'en ont nullement été incommodés. Des chiens ef des vautours ont dévoré également de la viande de moutons ayant reçu de très fortes doses d'arséniate (1 et $2 \mathrm{~g}$ ), apparemment sans dommage.

\section{I) DIFFUSION ACTUELLE DE L'ARSÉNIATE D'ÉTAIN DESTINÉ A L'USAGE VÉTÉRINAIRE}

Pour l'instant, le produit est utilisé uniquement en Chine populaire (1958) et en U. R. S. S. ou il tend d'ailleurs à devenir d'un emploi courant dans la lutte contre la moniéziose ovine, l'avitellinose et la thyzaniéziose. En 1955, 30.000 agneaux et 1.500 brebis ont été traités par Chubabriya (1958), et en Géorgie, 800.000 agneaux et 160.000 brebis au cours des années 1956-57.

Le ministère de l'Agriculture de I'U. R. S. S. a sanctionné en 1958 son utilisation et a chargé I'Institut de recherches et de médecine vétérinaire de Géorgie de préparer les quantités nécessaires : 20 tonnes ont été expédiées dans diverses républiques, ce qui représente environ 40 millions de doses. Pour 1959, on prévoit 30 tonnes et 60 millions de doses (Chubabriya, 1959).

Les Russes (Chubabriya, 1959) pratiquent le traitement prophylactique préimagal mis au point par Skrjabin ef Schultz (1934), traitement qui consiste à détruire le parasite avant que les signes de téniasis n'apparaissent dans le troupeau. Cetfe technique suppose des connaissances épidémiologiques précises et bien entendu, une solide enquête préalable.

Actuellement, les Russes (Chubabriya, 1959) effectuent, contre Moniezia exponsa, trois séries de traitements dans l'année, répartis ainsi :

Premier traitement : 5-10 jours avant la manifestation prévue de la maladie.

Second traitement : 25-30 jours plus tard.

Troisième traitement : $25-30$ jours après le deuxième.

En cas d'infestation massive, l'espacement des traitements sera de 10-15 jours au lieu de 25-30.

Paskalskaya (1959), de son côté, conseille quatre interventions :

la première : seconde quinzaine de mai ;

la seconde : un mois après ;

la troisième : fin juillet :

la quatrième : en septembre.

Au bout de deux ans, les exploitations sont considérées comme à peu près libres de moniéziose, mais elles demeurent encore quelque temps sous surveillance sanitaire.

\section{CONCLUSIONS}

1. - L'arséniate stanneux, $\mathrm{AsO}_{4} \mathrm{SnH}, 1 / 2 \mathrm{H}_{2} \mathrm{O}$, préparé à la Faculté de Pharmacie de Montpellier (Laboratoire de Pharmacie chimique), jouit d'une grande activité à l'égard de nombreux Anoplocephalidae de mouton tels que : Moniezia expansa, Moniezia benedeni, Stilesia globipunctata, Avitellina centripunctata ef Avitellina woodiandi. Ce corps rentre dans la catégorie des ténifuges polyvalents. Seul Stilesia hepatica échappe à son action.

c) La dose de $250 \mathrm{mg}$ par tête en une seule fois est particulièrement indiquée dans la lutte contre Moniezia expansa et Moniezia benedeni. La diète absolve de 20 heures, bien que fortement recommandable, n'est pas obligatoire. En ce cas, il est nécessaire de procéder «àsec» en supprimant toute distribution d'eau 12 heures avant ef 4 heures après le traitement.

c) La dose de $350 \mathrm{mg}$ par tête en une seule fois cssure la destruction de tous les Moniezia, de tous les Avitellina et de Stilesia globipunctata dans sa quasi-totalité. La diète absolue de 20 heures avant et de 4 heures après l'administration du produit est là strictement obligatoire.

c) La dose de $500 \mathrm{mg}$ par tête « à sec», sans diète, peut également être utilisée contre tous les Anoplocephalidae du mouton, à l'exception 
de Stilesia hepatica. Bien que très pratique, les risques qu'elle est susceptible de faire courir à l'animal en limitent l'intérêt.

II. - L'arséniate d'étain, dans tous les cas, doit être enfermé dans des capsules de gélatine rigaureusement dosées. Divers appareils permettent de faire parvenir celles-ci dans l'arrière-gorge de l'animal.

III. - Les conséquences du traitement à l'arséniate sont heureuses. II n'y a pas d'avortements de brebis pleines. L'augmentation de poids est spectaculaire. De plus, le produit provoque un « coup de fouet » brutal, de durée limitée, permettant à l'animal atteint de se rétablir dans un délai record.

IV. - La dose létale se situe autour de 2-2,25 g ce qui donne un coefficient chimio-thérapeutique de 8-9 (dose de $250 \mathrm{mg}$ ) et de 6 (350 mg). Les symptômes et les lésions observés sont ceux que l'on décrit classiquement lors d'intoxication arsenicale.

V. - Dans les pays tropicaux subsahariens, en raison des conditions extérieures défavorables (rickettsiose; helminthiases associées; alimentation et abreuvement déficients, etc...), l'arséniate d'étain à la dose de $500 \mathrm{mg}$ ef dans une moindre mesure à $350 \mathrm{mg}$ demande, de mai à novembre, d̀ être manipulé avec de grandes précautions en étudiant d'abord la résistance d'un petit lot d'ovins avant d'effectuer le traitement sur une échelle plus importante.

VI. - La toxicité pour l'homme des viandes provenant d'animaux traités à $250 \mathrm{mg}$ ef à $350 \mathrm{mg}$ est absolument nulle le cinquième ou le sixième jour après l'administration d'arséniate.

Les organes des ovins ayant reçu $250 \mathrm{mg}$ sont tous consommables 6 ou 7 jours après le traitement, sauf la rate. Il en va de même à la dose de $350 \mathrm{mg}$ : seuls la rate et l'intestin qui renferment une quantité d'arsenic supérieure au taux légal, seront rejetés de la consommation.

Laboratoire de pharmacie chimique de la Faculté de Montpellier

et

Service de parasitologie du Laboratoire de recherches vétérinaires de Farcha-Fort-Lamy (Tchad).

\section{BIBLIOGRAPHIE}

1. ADAMS, S. K. and HUGUES, L. E. (1955). Vet. Rec., 67, 733-5.

2. AKRAMOVSKI, M. N., EGOROV, Y. G. et BASHKIRTSEBA, E. V. (1958). - Veterinariyo, 34 (4), 43-4.

3. ANONYME (1958). - Chinese vet. J., 6, 242.

4. BARNES, J. M. et STONER, H. B. (1959). Pharm. Rev., II (2), 211-231.

5. BLAXTER, K. L., ALLCROFT and RUTH. (1950). - J. comp. Path., 60, 133.

6. BOEV, S. N. et ORLOV, N. P. (1958). Bull. Off. int. Epiz., 49 bis (11/12), 187-205.

7. CANAT (1956). - Bull. Soc. vét. prat., 40, 279.

8. CASTEL, P., HARANT, $H$. et GRAS, $G$. (1958). - Thérapie, 13 (5), 843-5.

9. CASTEL, P., HARANT, $H$. et GRAS, G. (1958). Théropie, 13 (5), 865-72.

10. CASTEL, P. et GRAS, G. (1959). - Rev. Path. gén., 59 (706), 327-30.

11. CASTEL, P., GRAS; G., GRABER, $M$. et CHHAY-HANCHENG (1960)... (en cours de publication).

12. CHUBABRIYA, I. T. (1955). - Trud. Gruzin. Nauchnoissled. Vet. Inst., II, 233-40.

13. CHUBABRIYA, I. T. (1957) - Veterinariya; 34 (12), 70-3.

14. CHUBABRIYA, I. T. (1959). - Bull. Off. int. Epiz., 49 bis (11/12), 211-31.

15. CHUBABRIYA, I. T. et GODERDZISHVILY, G. I. (1959). - Veterinariya, 36 (10), 34-6.

16. CRIBIER, J. (1921). - Thèse Doct. Pharm. Paris.

17. CRISTAU, M. B. (1958). - Ann. Pharm. fr. (11), 26-38.

18. EUZEBY, J. (1957). - Rev. Méd. vét., 108 (3), 178-84.

19. GARKAVI, B. L. (1956). - Veterinariya, 33 (9), 41-2.

20. GELOVANI, D. M., BERITASHVILI, T. P. ef LEONIDZE, L. A. (1958). - Sborn. Trud. Gruzin. Zooteckh. Vet. Inst., 10, 171-6. 
21. GRABER, M. (1957), - Rev. Elev. Méd. vét. Poys. trop., 10 (2), 119-28.

22. GRABER, M. (1959). - Symposium I.A.C.E. D. Nairobi (en cours de publication).

23. GRAS, G. (1958). - Thèse médicale. Montpellier.

24. GRIGORYAN, G. A., AKOPYAN, V. D., KHANBEGYAN, R. A., VEGAPETYAN, V. G. et AIVAZYAN, A. A. (1958). - Veterinariya, 35 (4), 43-4.

25. GRIFFON, H. et BUISSON, M. (1933). J. Pharm. Chim., 18, 422.

26. HOSKAM, E. G. (1958). - Tijd. V. Diergeneeskunde, 83, 413-23.

27. HUTCHINS. D. R. (1955). - Austr. vet. J., 31, 317-9.

28. LEWIS, E. F. and MEIKLE, J. C. (1956). Vet. Rec., 68, 98-9.

29. MC CULLOCH, E. C. and MC CLOY, J. E. (1941). — J. amer. Vet. Med. Ass., 99, 496-7.

30. MC CULLOCH, E. C. and St JOHN, J. L, (1940). - J. omer. Vet. Med. Ass., 98, 321-6.

31. MAMATELASHVILI, V. G., ABRAMISHVILI, E. S., MAKHASHVILI, A. S. et BERITASHVILI, K. P. (1958). - Sborn. Trud. Zooteckh. Vet. Inst., 10, 233-41.
32. NANOBASHVILI, V. I. (1959) - Veterinariya, $36(10), 56-7$.

33. OVENSTONE, T. C. ef KENYON, C. (1955). - Analyst., 80, 566-67.

34. PASKALSKAYA, M. Y. (1959). - Veterinariya, $36(10), 21-3$.

35. PRASAD, M. and DESAI, D. M. (1939). S. Ind. Chem. Soc., 117, 26.

36. REMY, R. (1956). - Deutsch. tierárz. Wochensch., 63, 385-88 ef 405-9.

37. SCHOBERL, A. (1958) - Deutsch. tierârz. Wochensch., 65, 235-9.

38. SEDDON, J. R. and RAMSAY, A. A. (1933). - N. S. Wales. Dept. Agr. Vet. Res. Rept., 6 (3), 113.

39. SIGETO HAMADA (1949). - J. Agr. Chem. Soc. Japon., 23, 13-5.

40. SKJABIN, K. I. et SCHULTZ (1934) - Bull. Off. int. Epiz., 7 (1), 354-78.

41. SIVADIIAN, J. (1946). - C. R. Soc. Biol., I40, 845.

42. TALBERT, T. J. and TAYLOE, W. L. (1933). - Missouri State Res. Bull., 133.

43. TCHOUBABRIE, I. T. - Voir: Chubabriya.

44. ULYANOV, S. D. (1957). - Veterinariya, 34 (5), 32-5.

\section{SUMMARY}

\section{The action of arseniate of tin on ovine cestodes}

Arseniate of tin $\left(\mathrm{AsO}_{4} \mathrm{HSn}, 1 / 2 \mathrm{H}_{2} \mathrm{O}\right)$ is completely efficaceous in the destruction of $M$. expansa, M. benedeni, S. globipunctata and A. centripunctata of sheep. Two types of dosing are required following deprication of water for 12 hours before and 4 hours after treatment. One dose of $250 \mathrm{mgs}$. per head in the case of $M$. expansa and $350 \mathrm{mgs}$. per head in thecase of infestation with Anoplocephalidae spp. with the exclusion of S. hepatica. In the latter case complete starvation for 20 hours before and 4 hours after administration of the drug is essential.

This drug does not cause abortion in pregnant ewes; the increase in weight is very rapid and an infected flock is reconditioned in record time.

In a tropical environment this product is to be handled with extreme care, particularly in the larger dosage during the period May to November on account of the unfavourable climatic conditions, but in the dry season from November to April, its presents no danger.

The lethal dose is around 2 to $2.5 \mathrm{gms}$. giving a chemotherapeutic coefficient of $8-9$ with the smaller dose and of 6 with the larger.

The meat of animals treated with this product is fit for consumption in 5-6 days after treatment. 


\section{RESUMEN}

Acción del arsenlato de estan̂o sobre diversos cestodes del carnero.

El arseniato de estaño AsO4 $\mathrm{HSn}^{4}, 1 / 2 \mathrm{H}^{2} \mathrm{O}$ asegura la destrucción de Moniezia expansa. Moniezia benedeni, Stilesia globipunctata y Avitellina centripunctata del carnero. Dos dosis son preconizadas : $250 \mathrm{mgr}$ por cabeza en una solva vez contra Moniezio expansa, suprimiendo completamente toda distribución de agua 12 horas antes y 4 horas después del tratamiento, y $350 \mathrm{mgr}$ por cabeza en una sola vez contra todos los Anoplocefalidae, excepción de Stilesia hepatica. En este caso, la dieta absoluta es de rigor 20 horas antes y 4 horas despues de la administracion del producto.

El arseniato de estaño no ocasiona abortos en las ovejas preñadas. El aumento de peso es muy rápido y el rebaño infectado se restablece en un tiempo record.

En medio tropical, el producto deberá ser manejado con precauciones extremas (dosis de $350 \mathrm{mgr}$ sobre todo) de mayo a noviembre, a causa de condiciones exteriores desfavorables (estado mediocre de los animales, rickettsiosis, etc...). De noviembre a abril no presenta ningún peligro.

La dosis letal se sitúa abrededor de 2 à $2,5 \mathrm{gr}$, lo que proporciona un coeficiente quimio terapéutico de 8-9 (250 mgr) ó de 6 (350 mgr).

La carne de los animales tratados con el arseniato puede ser consumida de 5 à 6 días después del final del tratamiento. 